\title{
Revealing the role of local stress on the depolarization of BNT-BT-based relaxors
}

\author{
Julia Glaum, ${ }^{1,2,{ }^{*}}$ Yooun Heo, ${ }^{2}$ Matias Acosta, ${ }^{3}$ Pankaj Sharma, ${ }^{2}$ Jan Seidel, ${ }^{2}$ and Manuel Hinterstein ${ }^{2,4}$ \\ ${ }^{1}$ Department of Materials Science and Engineering, Norwegian University of Science and Technology NTNU, 7491 Trondheim, Norway \\ ${ }^{2}$ School of Materials Science and Engineering, UNSW Sydney, Sydney, New South Wales 2052, Australia \\ ${ }^{3}$ Department of Geo- and Materials Science, Technische Universität Darmstadt, 64287 Darmstadt, Germany \\ ${ }^{4}$ Institute for Applied Materials, Karlsruhe Institute for Technology, 76131 Karlsruhe, Germany
}

(Received 20 February 2019; published 15 May 2019)

\begin{abstract}
Canonical relaxors exhibit an electric-field-induced phase transition between a macroscopically nonpolar and polar phase that can be tuned from being stable at low temperature to being reversible at high temperature. The reversibility of this phase change determines the electromechanical performance and large strains can be achieved if the polar phase is intrinsically unstable. This paper is on the thermal depolarization characteristics of a BNT-BT-based multiphase relaxor ceramic observed through the transition temperature from field-induced polar to nonpolar state. It is shown that the progress of detexturization strongly depends on the crystallographic phase. In the more susceptible phase, it becomes significant about $40{ }^{\circ} \mathrm{C}$ below the macroscopically observed transition temperature. Additionally, the surface domain structure vanishes at lower temperatures than expected from both dielectric and structural measurements. The development of strong interfacial stresses aiding depolarization, and a mismatch in chemical pressure between surface and bulk, are discussed as the origins for the observed effects. Tailoring of interfacial stresses through chemical adaption of crystallographic phase fractions opens up a pathway to optimize the strain performance of actuator materials and can become a useful tool to stabilize metastable crystallographic phases as well as for property tuning in piezotronics, Mott insulators and multiferroics.
\end{abstract}

DOI: 10.1103/PhysRevMaterials.3.054406

\section{INTRODUCTION}

The unique properties displayed by canonical relaxor systems, such as the electric-field-induced change between a pseudocubic and a long-range polar state [1-5], the associated huge unipolar strain [6,7], and the phase-transition-mediated polarization reversal mechanism [8], make these materials fascinating and challenging objects of study in materials science research.

Especially, the peculiar differences of the dielectric characteristics in the electrically poled and unpoled state were the focus of many studies during the last few decades [9-14]. The unpoled state is determined by the presence of local polar behavior. In this state, the electric dipoles are not correlated [15], leading to distinct dispersive characteristics of the dielectric properties. Electric poling can enforce correlated behavior, such that a ferroelectric long-range order is established. The stability of this ferroelectric order in the field-free state is determined by chemical composition as well as prevalent temperature. For a specific composition, there exist different threshold temperatures: $T_{\mathrm{F}-\mathrm{R}}$, marking the transition from correlated to uncorrelated behavior-or from ferroelectric to relaxor characteristics $[8,16,17]$ and $T_{\mathrm{d}}$, marking the loss of preferred domain orientation [18].

\footnotetext{
*julia.glaum@ntnu.no

Published by the American Physical Society under the terms of the Creative Commons Attribution 4.0 International license. Further distribution of this work must maintain attribution to the author(s) and the published article's title, journal citation, and DOI.
}

The instability of the induced ferroelectric state is the main origin for the appearance of high unipolar strains in relaxor-ferroelectric materials [3,19]. It is known that the transition temperature $T_{\mathrm{F}-\mathrm{R}}$ can be tuned by chemical doping [20], introducing a higher intrinsic degree of disorder into the system. This effect can as well be achieved by the intentional design of ferroelectric/relaxor core-shell composites [4,21,22] or by controlled thermal treatment, such as quenching from high temperatures [23]. Application of mechanical stress is another means to tune $T_{\mathrm{F}-\mathrm{R}}$ and with this to favor the longrange polar state over the pseudocubic state [24]. The close link between mechanical stress state and prevalent crystallographic phase is not only fundamental for relaxor/ferroelectric systems [25,26], but as well for many other material systems, e.g., structural ceramics [27], metals [28], and various composites [29].

Many relaxor-ferroelectric ceramics exhibiting high actuating performance are not only chemically rather complex, but are also located in the morphotropic phase region leading to the appearance of multiple crystallographic phases even within single grains [30]. One system exhibiting such an "intrinsic core-shell structure" of a polar minority phase embedded into a polar majority phase is $\left(\mathrm{Bi}_{1 / 2} \mathrm{Na}_{1 / 2}\right) \mathrm{TiO}_{3}-\mathrm{BaTiO}_{3}$ (BNT-BT). For the morphotropic composition BNT-6BT the majority phase was reported to be of rhombohedral nature, while the minority phase is tetragonal [5]. Its ability to take on different phases simultaneously and to change the phase ratio to adapt to external stimuli [5,24] is the main origin for its exceptional electromechanical performance. Across the phase boundary at around $6 \% \mathrm{BT}$ the pseudocubic relaxor phase is stable in the unpoled state, whereas the electric-field-induced 
phase composition of distorted ferroelectric phases strongly varies across the phase boundary [5]. High actuating strains, however, are only achieved if the reversibility of the relaxorferroelectric transition is exploited, e.g., by operating the material close to its $T_{\mathrm{F}-\mathrm{R}}$. The thermal evolution of the material properties gives insight into the mechanisms governing the decay from correlated to uncorrelated behavior, and detailed studies on the development of dielectric properties [31], domain patterns [32], and structural characteristics [33,34] are available.

In this paper we focus on the multiphase nature of the $\left(\mathrm{Bi}_{1 / 2} \mathrm{Na}_{1 / 2}\right) \mathrm{TiO}_{3}-\mathrm{BaTiO}_{3}$ system and the evolution of the polar phases upon approaching and passing of $T_{\mathrm{F}-\mathrm{R}}$ with increasing temperature. While the majority phase stays stable up to $T_{\mathrm{F}-\mathrm{R}}$ in terms of texture and unit-cell dimensions, a gradual detexturization of the minority phase is observed over the whole temperature range. In contrast to that, the surface domain structure decays already at significantly lower temperatures. Interfacial stresses developing between majority and minority phases as well as differences in local stress state between surface and bulk are discussed as driving factors of the phase-transition dynamics. Tailoring of the interfacial stress state in multiphase relaxor-based materials can be a tool to optimize their actuating performance.

\section{EXPERIMENTAL PROCEDURE}

0.94 $\left(\mathrm{Bi}_{1 / 2} \mathrm{Na}_{1 / 2}\right) \mathrm{TiO}_{3}-0.06 \mathrm{Ba}\left(\mathrm{Zr}_{0.05} \mathrm{Ti}_{0.95}\right) \mathrm{O}_{3} \quad$ ceramics were prepared via solid-state processing. A detailed description of the processing routine can be found elsewhere [20,35].

Measurements of strain $S$ and piezoelectric coefficient $d_{33}$ were performed using a commercial TF2000 system (AixACCT, Aachen, Germany). For the electric measurements, disk-shaped samples of about 7-mm diameter and 1-mm thickness were polished to $15-\mu \mathrm{m}$ finish and annealed at $300{ }^{\circ} \mathrm{C}$. Sputtered gold was applied as electrodes. The strain was determined at a frequency of $f=1 \mathrm{~Hz}$. The measurement parameters for the remanent $d_{33}$ were $f=1 \mathrm{kHz}$ and $E_{\mathrm{ac}}=$ $2.6 \mathrm{~V} / \mathrm{mm}$. To ensure that the samples were homogeneously polarized, the same setup was used to pole the samples before the piezoresponse force microscopy (PFM) studies. In this case, the samples were polished to $1 / 4-\mu \mathrm{m}$ finish using diamond paste. Air-dried silver electrodes were applied for poling and were washed off with ethanol before the PFM scans. The samples were poled using a triangular waveform with a frequency of $f=1 \mathrm{~Hz}$ and a maximum electric field of $E=4.5 \mathrm{kV} / \mathrm{mm}$.

Temperature- and frequency-dependent permittivity was determined using an Alpha A High Performance Frequency Analyzer coupled to a Novotherm furnace (Novocontrol Technologies, Montabaur, Germany). The excitation voltage chosen was $1 \mathrm{~V}_{\text {rms }}$ corresponding to $1.15 \mathrm{~V} / \mathrm{mm}$.

The temperature-dependent piezoresponse force microscopy study was conducted with an AIST-NT Smart SPM1000 system in air. Mikromasch Pt-coated probes (NSC35 ) were used for imaging. Neutron-diffraction measurements were taken at the Wombat diffractometer at the Bragg Institute, ANSTO, Sydney, Australia [36]. The samples used were of matchstick shape with a base of $3.5 \times 3.5 \mathrm{~mm}$ and a length of about $25 \mathrm{~mm}$. The samples were polished to a $15-\mu \mathrm{m}$ finish and air-dried silver electrodes were applied to two opposing sides of $3.5 \times 25 \mathrm{~mm}$. Placed already in the sample holder, but prior to measurement, one bipolar electric cycle with a frequency of $1 \mathrm{~Hz}$ and a maximum electric field of $4.5 \mathrm{kV} / \mathrm{mm}$ was applied to the sample using a Trek 20/20 high-voltage amplifier (TREK, INC., Medina, NY). Texture measurements were taken in the remanent state by recording multiple diffraction exposures as the sample was rotated in $15^{\circ}$ steps through $180^{\circ}$. Texture measurements were conducted at several temperature steps upon heating from room temperature to $100{ }^{\circ} \mathrm{C}$. Details of the sample environment and the measurement method can be found elsewhere [19,37]. Strain and phase analysis was carried out using the software package MAUD (Materials Analysis Using Diffraction) [38] and a detailed description can be found elsewhere [39].

For microstructural analysis a sample was polished to a $1-\mu \mathrm{m}$ finish and subsequently thermally annealed at $970{ }^{\circ} \mathrm{C}$ for $5 \mathrm{~min}$. Micrographs were taken using a Zeiss Supra 55VP scanning electron microscope (Carl Zeiss AG, Oberkochen, Germany). Supplemental Material, Fig. S1 shows a micrograph of the thermally etched sample surface [40].

\section{RESULTS AND DISCUSSION}

Measurements were conducted on dense bulk ceramics (Fig. S1 [40]) of composition 0.94( $\left.\mathrm{Bi}_{1 / 2} \mathrm{Na}_{1 / 2}\right)$ $\mathrm{TiO}_{3}-0.06 \mathrm{Ba}\left(\mathrm{Zr}_{0.05} \mathrm{Ti}_{0.95}\right) \mathrm{O}_{3}$, with the $\mathrm{Zr}$ dopant taking influence on the transition temperatures compared to undoped 94BNT-6BT, but not the general relaxor-ferroelectric characteristics [20].

The temperature-dependent strain hysteresis loops depicted in Fig. 1 clearly show a change in the mechanism determining the strain output. For temperatures up to $70{ }^{\circ} \mathrm{C}$ a typical ferroelectric "butterfly" shape of the hysteresis is observed, which starts to deform around $80{ }^{\circ} \mathrm{C}$ and transforms into a "sproutlike" shape with further increasing temperature. The latter is signified by the lack of remanent strain as well as unipolar strain values comparable to commercially used $\mathrm{Pb}(\mathrm{Zr}, \mathrm{Ti}) \mathrm{O}_{3}$-based ceramics [7]. The change in hysteresis shape is related to the material-specific transition temperature $T_{\mathrm{F}-\mathrm{R}}$, indicating the loss of ferroelectric long-range order.

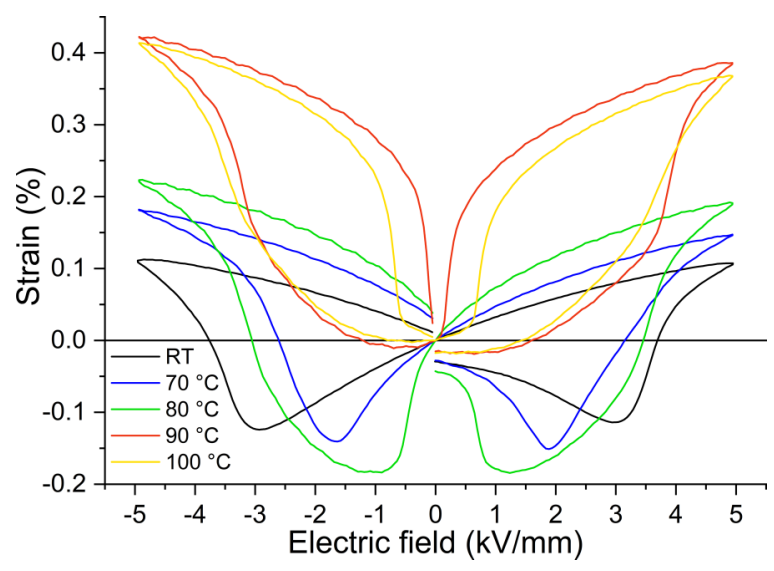

FIG. 1. Temperature-dependent strain hysteresis loops. 


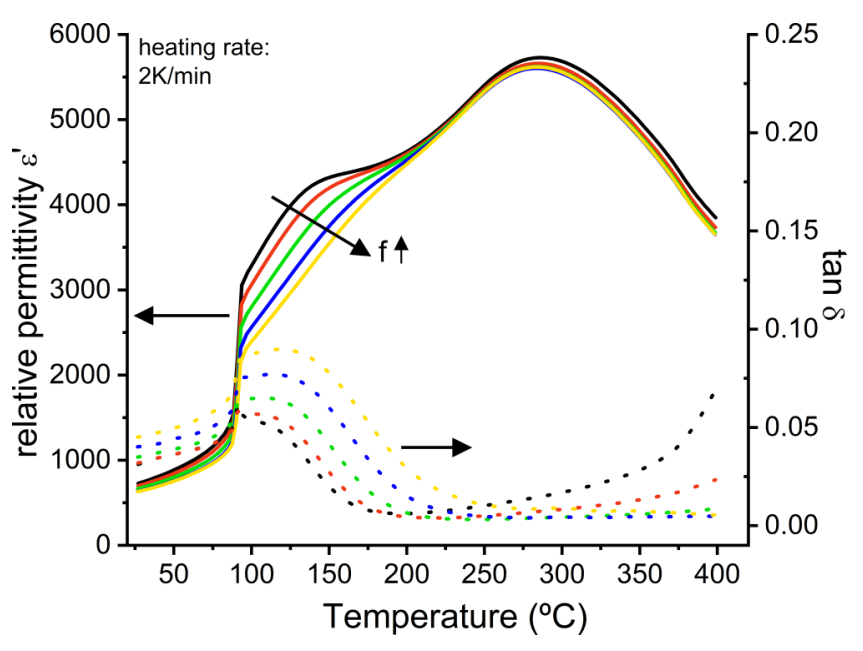

FIG. 2. Temperature- and frequency-dependent permittivity and $\tan \delta$, heating rate of $2 \mathrm{~K} / \mathrm{min}, 100 \mathrm{~Hz}<f<0.3 \mathrm{MHz}$.

Figure 2 presents the temperature- and frequencydependent permittivity $\varepsilon^{\prime}$ and $\tan \delta$ as measured on an electrically prepoled sample. As different heating rates were used in the experiments presented in this study, the temperaturedependent permittivity was recorded for the two extreme cases, namely $2 \mathrm{~K} / \mathrm{min}$ (Fig. 2) and $0.5 \mathrm{~K} / \mathrm{min}$ (Fig. S2 [40]). For both heating rates, no significant difference of $T_{\mathrm{F}-\mathrm{R}}$ could be observed, making the results obtained by the different methods presented comparable. The nondispersive behavior at temperatures below $90{ }^{\circ} \mathrm{C}$ changes to strong dispersive characteristics up to about $200{ }^{\circ} \mathrm{C}$, which indicates the loss of ferroelectric long-range order. Both the dispersive characteristics and the maximum permittivity value around $280^{\circ} \mathrm{C}$ are in line with other reports on this material system $[31,34,41]$. The transition from ferroelectric to relaxor state occurred at $91{ }^{\circ} \mathrm{C}$, while the depolarization temperature $T_{\mathrm{d}}$, at which dipole correlation still remains but the preferred domain orientation decays [18], was found to be $87^{\circ} \mathrm{C}$ (Fig. S3 [40]).

To investigate the impact of temperature on the domain pattern, PFM studies were conducted. The ceramic sample was poled ex situ to achieve a fully poled bulk and then transferred to the PFM setup for temperature-dependent measurements. Figure 3(a) shows vertical-amplitude PFM images after local electrical poling with different voltages at room temperature, where each bias voltage was applied in the marked box areas (dotted lines). Relatively low voltages around 9-10 V lead to domain changes and likely surface poling, i.e., not through the whole depth of the bulk ceramic sample. To scan the domain pattern as it was introduced during ex situ poling, care was taken to apply only small biases below $1.5 \mathrm{~V}$ to avoid any additional poling during the measurement. Topography measurements showed that samples were rather smooth with a root-mean-square surface roughness of around $2 \mathrm{~nm}$ [Fig. 3(b)].

Figures 3(c) and 3(d) show images of the lateral phase taken at 25 and $75^{\circ} \mathrm{C}$, respectively. The ex situ poling process leads to a well-poled sample at room temperature with a stable long-range domain structure as observed in Fig. 3(c). In the as-prepared state, an irregular domain microstructure exists with surface domain sizes around hundreds of nanometers in both the acquired in-plane and out-of-plane piezoresponse. However, already at $75^{\circ} \mathrm{C}$, which is about $10^{\circ} \mathrm{C}$ below $T_{\mathrm{d}}$, the overall large-scale domain structure is replaced by a grainy contrast and only a few larger domains remain [Fig. 3(d)]. Similar to previous in situ poling studies [42], the domain patterns observed at the sample surface decay at significantly lower temperatures than expected from the bulk piezoelectric and dielectric observations in Figs. 1 and 2. As the sample was not just locally poled but had been subjected to the poling field over the whole sample volume, a more fundamental mechanism than the previously proposed incomplete poling of the sample seems to govern this effect.

To further explore the development of domain texture, temperature-dependent neutron-diffraction measurements were conducted (Fig. 4). Similar to the temperature dependency of the domain patterns, distinct changes of the crystallographic structure are expected upon heating through $T_{\mathrm{d}}$ and $T_{\mathrm{F}-\mathrm{R}}$. From the presence of the one-half $311_{\mathrm{C}}$ superlattice reflection [Fig. 4(a)] as well as the apparent peak splitting of the $111_{\mathrm{C}}$ and $200_{\mathrm{C}}$ reflections [Figs. 4(b) and 4(c)] it can be concluded that the poled state exhibits a tetragonal/rhombohedral phase mixture, which previously was

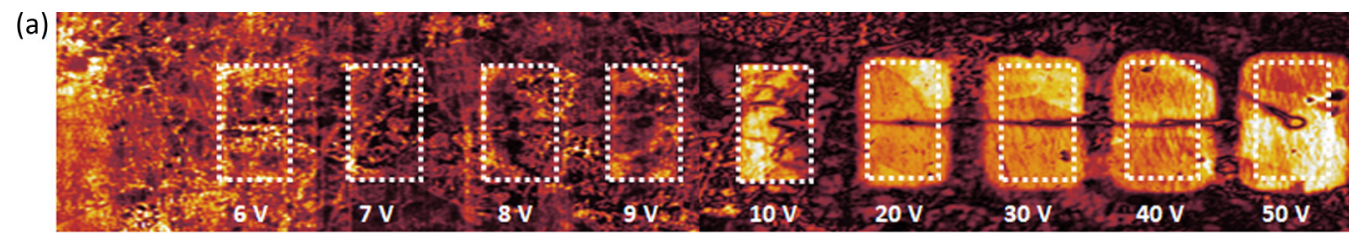

(b)

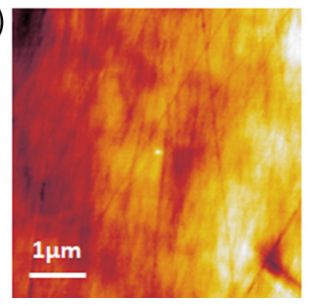

(c)

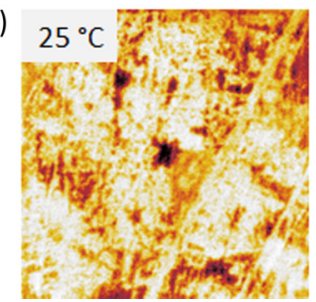

(d)

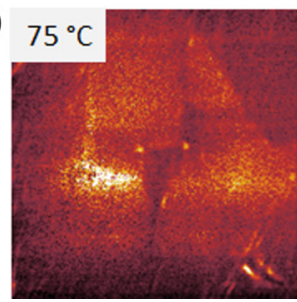

FIG. 3. Temperature-dependent PFM measurements.( a) Vertical-amplitude PFM images after local electrical poling with different voltages at room temperature, where each bias voltage was applied in the box areas (dotted lines). b) Topography scan with a root-mean surface roughness of around $2 \mathrm{~nm}$, lateral $\mathrm{PFM}$ phase at (c) $25^{\circ} \mathrm{C}$, (d) $75^{\circ} \mathrm{C}$. 


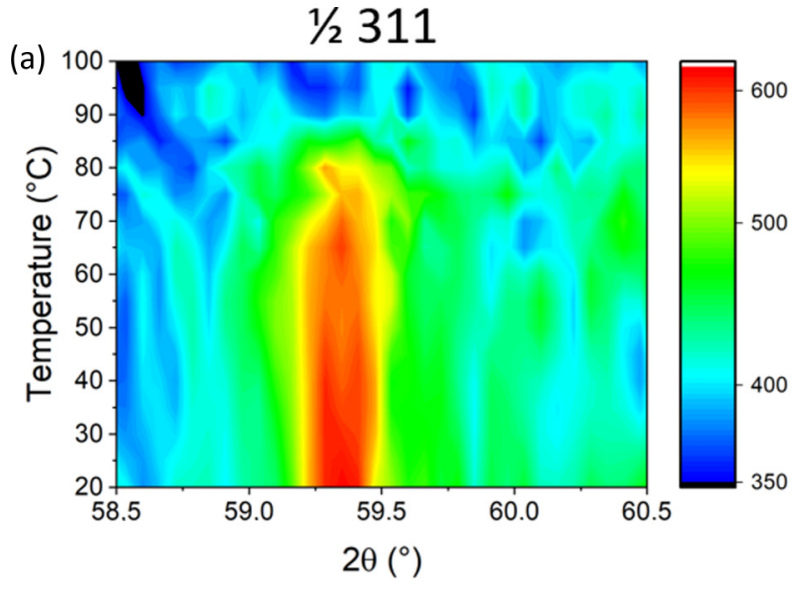

111
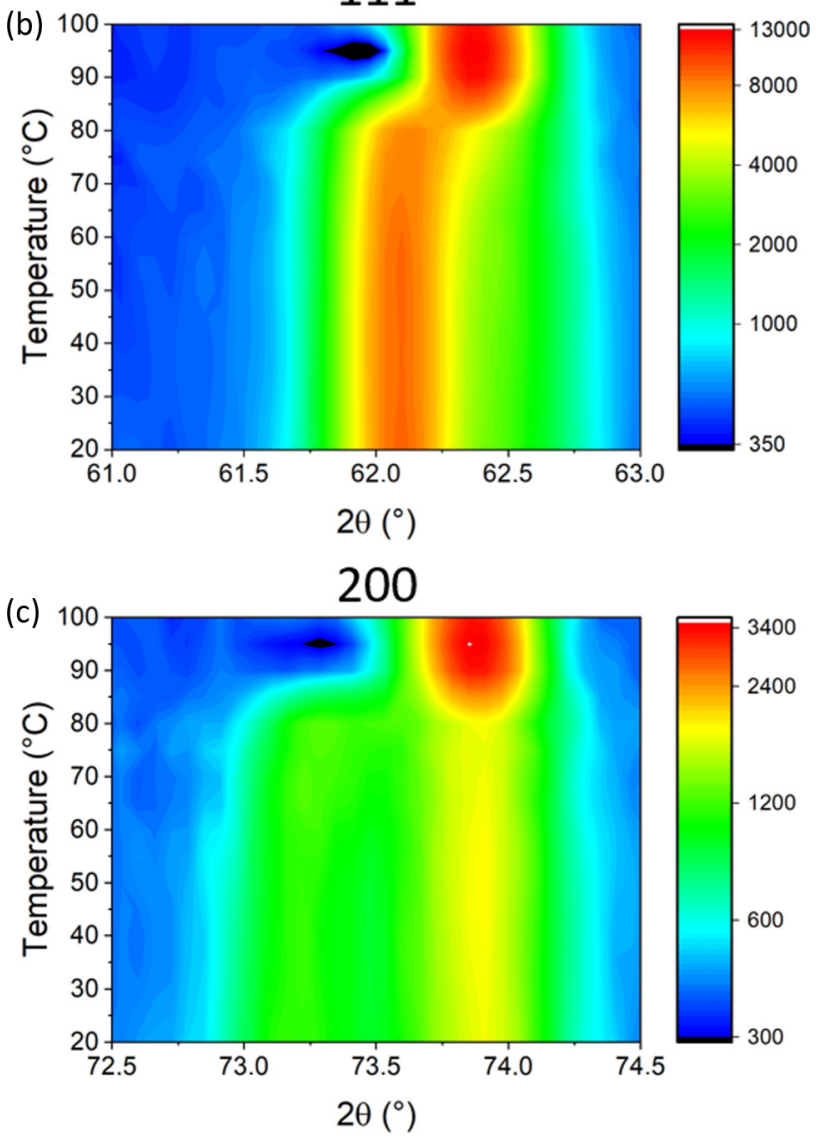

FIG. 4. Contour plots showing the development of the $1 / 2311_{c}$, $111_{c}$, and $200_{c}$ reflections taken by neutron diffraction upon increasing temperature. The sample was oriented such that the electric-field vector upon poling was located between the scattering vectors of the $111_{c}$ and $200_{c}$ reflections. The scales were adapted for each reflection for improved visualization.

identified to consist of tetragonal $P 4 \mathrm{~mm}$ and rhombohedral $R 3 c$ [43]. The tetragonal phase is the minority phase with about 20\% phase content at room temperature. For BNT-BTbased systems there are a number of reports showing that different phases appear as locally clearly separated regions, often sharing a single grain [43-45].
With increasing temperature, the polar characteristics are lost between 80 and $85{ }^{\circ} \mathrm{C}$, which is slightly below $T_{\mathrm{F}-\mathrm{R}}=$ $91{ }^{\circ} \mathrm{C}$ extracted from Fig. 2. For higher temperatures the average structure appears to be cubic in agreement with previous reports $[8,46]$.

The temperature-dependent development of the texture for each phase can be followed with the pole figure densities (PFDs) $f(h k l)$, generated from the orientation distribution function (given in multiples of random distribution) in Fig. 5. Both phases exhibit very high ferroelastic PFD values in poling direction, highlighting that the electric-field-induced phase transformations allow a high degree of alignment along the electric-field vector. The morphotropic character of the material allows precipitation of either the tetragonal or the rhombohedral phase from the pseudocubic state, depending on which phase is favored by the grain orientation. Similar characteristics have been reported for $\mathrm{Bi}_{1 / 2} \mathrm{Na}_{1 / 2} \mathrm{TiO}_{3}-\mathrm{SrTiO}_{3}$ core-shell compositions [4].

With increasing temperature, the textures of the rhombohedral and tetragonal phase develop quite differently (Fig. 5). While the former stays unchanged up to $85{ }^{\circ} \mathrm{C}$ and vanishes for higher temperatures resembling the macroscopic depoling characteristics in Fig. S3 [40], the changes for the latter are gradual and the loss of texture becomes already apparent at $40{ }^{\circ} \mathrm{C}$. This is also reflected in the domain switching strain $S_{\mathrm{D}}$ [Fig. 6(a)].

While the tetragonal domain switching strain $S_{\mathrm{D}, \mathrm{T}}$ gradually decreases with temperature from a high value of $0.5 \%$ at room temperature, the rhombohedral domain switching strain $S_{\mathrm{D}, \mathrm{R}}$ stays almost constant between 0.3 and $0.2 \%$ over the whole temperature range.

The reason for the slight decrease in $S_{\mathrm{D}, \mathrm{R}}$ is the change in lattice distortion $\eta$ (Fig. S4 [40]), which is an important factor for calculating the domain switching strain from the PFDs [38]:

$$
S_{\mathrm{D}}=\eta \frac{1}{2 \pi} \int_{\alpha=0}^{\pi / 2}\left[\Delta f(h k l) \cdot \cos ^{2} \alpha\right](\sin \alpha) \mathrm{d} \alpha,
$$

Since the rhombohedral lattice distortion $\eta_{\mathrm{R}}$ is significantly lower than the tetragonal $\eta_{\mathrm{T}}$, the rhombohedral domain switching strain $S_{\mathrm{D}, \mathrm{R}}$ is also lower than the tetragonal one, $S_{\mathrm{D}, \mathrm{T}}$. Figures 6(a)-6(c) show that the tetragonal extrinsic and intrinsic contributions, namely domain switching strain $S_{\mathrm{D}, \mathrm{T}}$ and lattice strain $S_{\mathrm{L}, \mathrm{T}}$, respectively, predominantly determine the overall strain response $S$. The rhombohedral lattice strain $S_{\mathrm{L}, \mathrm{R}}$ is negligible over the whole temperature range. The high-temperature stability of the rhombohedral, ferroelastic domain structure is a consequence of the temperature stability of the rhombohedral phase fraction, which is around $80 \%$ (Fig. S5 [40]).

A system exhibiting multiple phases simultaneously is per $s e$ very susceptible to external stimuli. Specifically, changes in phase fraction induced by electric fields and mechanical loads are facilitated by the decrease of lattice distortion towards the phase boundary. The interplay of coexisting phases as well as the local state of stress and electric field at their interface will strongly determine the stability of the system. The external stimulus in the present study is the change in temperature. Thermal expansion and randomization of the highly aligned 

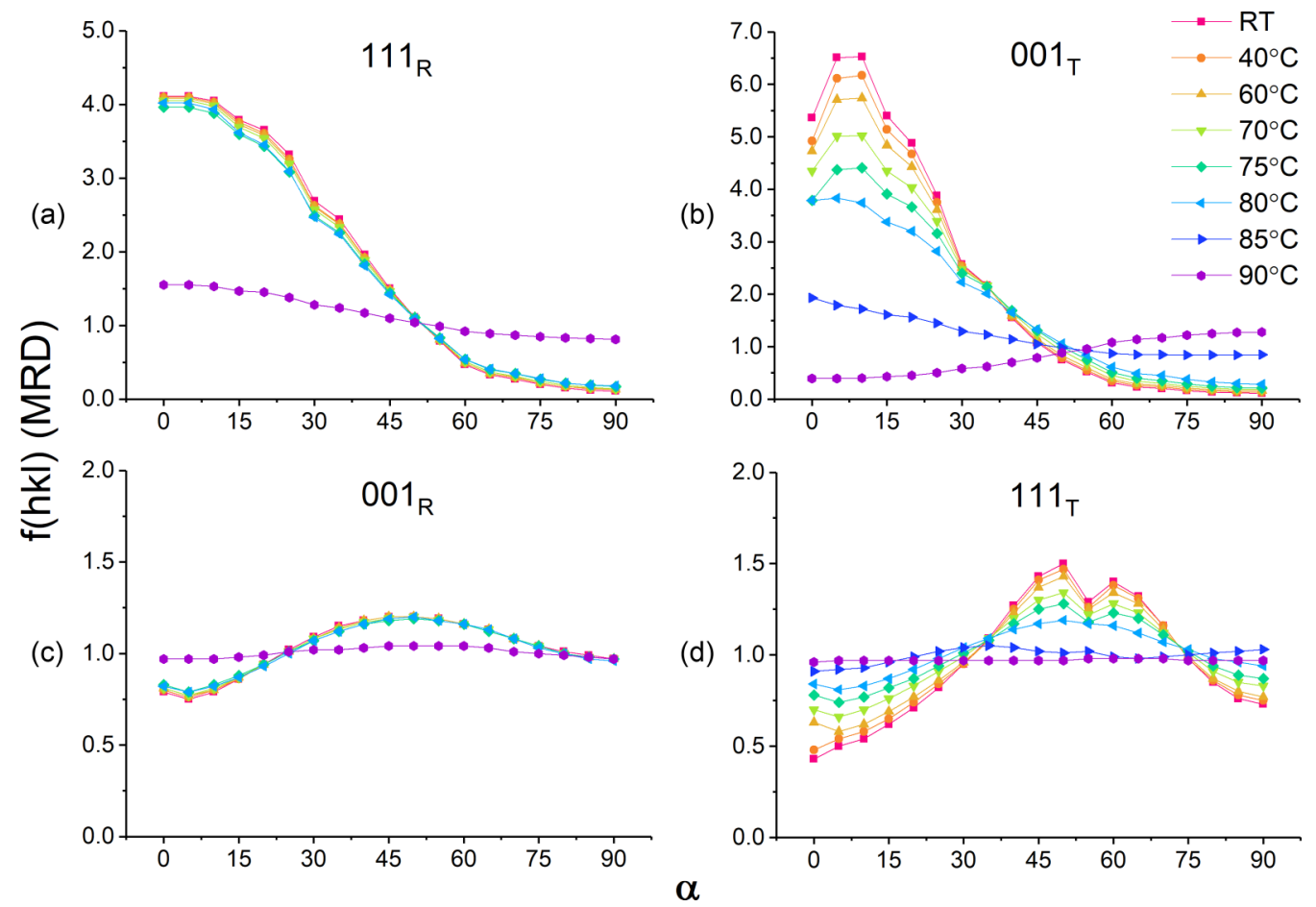

FIG. 5. Temperature-dependent pole figure densities for both the rhombohedral (a), (c) and tetragonal (b), (d) contribution to the 111 and 001 reflections.

domain state are, therefore, factors to consider in explaining the different developments in phase texture.

Thermal expansion coefficients of the different phases can be calculated from the temperature-dependent lattice parameters (Fig. S6 [40]) and a rough approximation of the developing interfacial stresses can be obtained using a Young's modulus of $128 \mathrm{GPa}$ as reported for bulk BNT-6BT ceramics [41], keeping in mind that individual phase moduli in morphotropic systems can vary considerably on the nanoscale $[47,48]$. Table I summarizes the thermal expansion coefficients $\alpha$ in the $a$ - and $c$ direction and the corresponding stresses, developed at the interface between rhombohedral and tetragonal phases. Both phases show anisotropic thermal characteristics, with expansion in the $a$ direction and contraction in the $c$ direction upon heating. Depending on the orientation of the neighboring phases towards each other, the anisotropic characteristics lead to differences in the interfacial stress.

Figure 7 provides a sketch of different orientation arrangements and the corresponding stresses at the interface. For an unpoled sample the stress levels as calculated to appear at the interface between the rhombohedral and tetragonal phases would lead to mostly elastic response [49]. However, the response of poled ferroelectric materials to mechanical pressure significantly differs from the unpoled case, as the aligned domain structure in the remanent state is metastable and domain switching can occur more easily. The coercive stress of poled BNT-BT ceramics under uniaxial compression was found to increase from $-162 \mathrm{MPa}$ for a tetragonal composition to $-253 \mathrm{MPa}$ for a rhombohedral composition [50]. This highlights that stress levels, as induced at the phase interface due to thermal expansion, are sufficient to initialize domain switching and that the tetragonal minority phase is intrinsically more susceptible to the local stress state. This can explain the onset of detexturization of the tetragonal phase at temperatures much below $T_{\mathrm{d}}$ and $T_{\mathrm{F}-\mathrm{R}}$.

The loss of domain texture at temperatures below $T_{\mathrm{d}}$ as observed in the PFM images (Fig. 3) seems to correspond with the loss in crystallographic texture observed in the neutrondiffraction studies. However, the reduction of crystallographic texture is determined by the tetragonal minority phase (Fig. 5), which makes up only $20 \%$ of the phase content, whereas the loss in surface domain texture is observed in all regions investigated. Therefore, it seems that detexturization is governed by different factors in the bulk and at the surface. The surface characteristics are signified by a lower transition temperature $T_{\mathrm{d}}^{*}$ compared to the characteristics of the bulk. Surface domain texture is lost already at $75{ }^{\circ} \mathrm{C}$, whereas crystallographic texture of the bulk is stable up to $85^{\circ} \mathrm{C}$. For bulk samples a shift in transition temperatures can be induced both by the application of an electric field [51] or a mechanical load $[52,53]$, indicating that in the present case heterogeneous local electrical or strain fields might be the cause for the observed differences between bulk and surface.

In the present study, the whole sample was electrically poled at the same conditions for all experiments. However, the electric boundary conditions during heating differed, as the electrodes were kept on for the dielectric and the neutron-diffraction measurements, whereas the electrode on the probed surface had to be removed for the PFM experiment. This could potentially lead to an increased screening length in the PFM measurements compared to the other experiments and a decrease of the transition temperatures, as indicated by calculations on ferroelectric $\mathrm{PbTiO}_{3}$ thin films [54]. These calculations show, however, that the shift in the characteristic temperatures becomes less pronounced with increasing film thickness and should be negligible for bulk samples. 

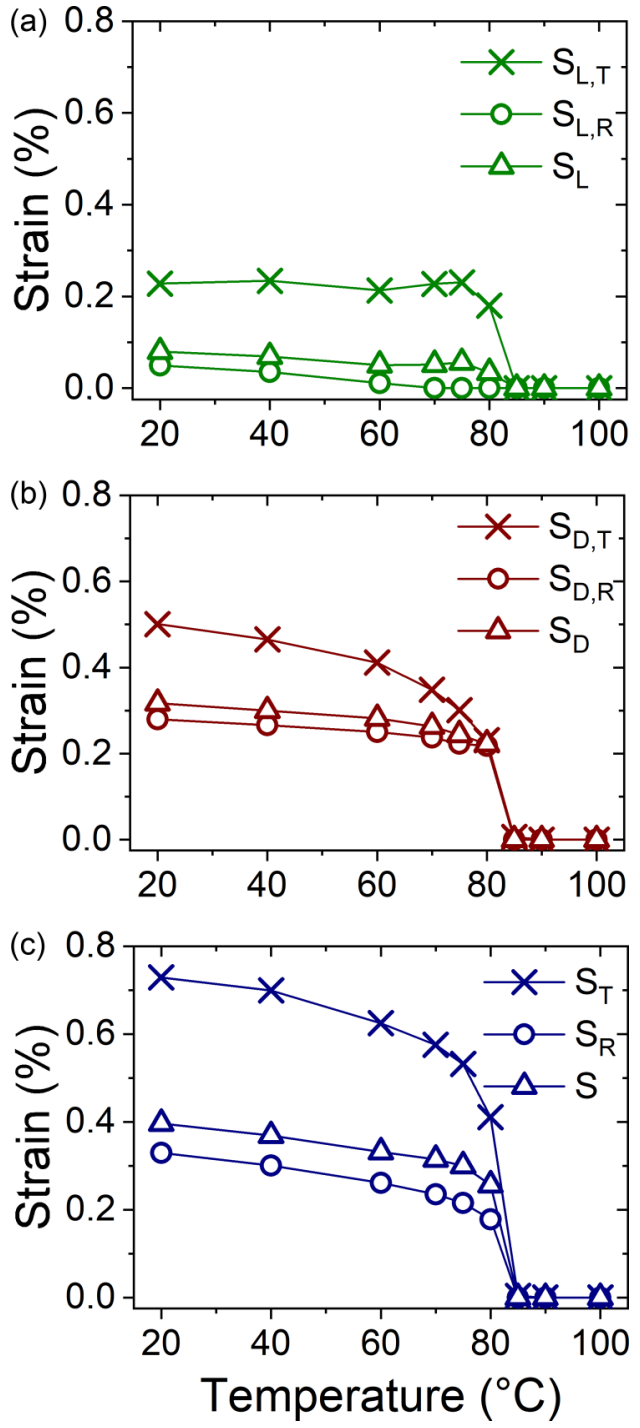

FIG. 6. Temperature dependence of (a) lattice strains $S_{\mathrm{L}}$, (b) domain strains $S_{\mathrm{D}}$, and (c) total strains $S$ for both the tetragonal and rhombohedral phase (subscript " $T$ " and "R," respectively) as observed when heating a prepoled sample up to $100^{\circ} \mathrm{C}$.

Besides heterogeneous electric fields, variations in the mechanical stress state could be the source of the differences in temperature-dependent response. Application of hydrostatic compressive stress has been proven to strongly decrease the characteristic temperatures of various relaxor materials. Related to the observations in the present study, this could indicate that the sample bulk experiences tensile stress that relaxes towards the sample surface. Indeed, it was reported that Ba doping of BNT introduces a tensile chemical pressure (about 2.5 GPa for BNT-11BT [55]). This is significantly higher than

TABLE I. Thermal expansion coefficients $\alpha$ of both rhombohedral and tetragonal phase, corresponding stresses $\sigma$.

\begin{tabular}{lcccc}
\hline \hline & $\alpha_{\text {tetra }}\left(\mathrm{K}^{-1}\right)$ & $\sigma_{\text {tetra }}(\mathrm{MPa})$ & $\alpha_{\text {rhomb }}\left(\mathrm{K}^{-1}\right)$ & $\sigma_{\text {rhomb }}(\mathrm{MPa})$ \\
\hline$a$ direction & $2.122 \times 10^{-5}$ & 163 & $1.319 \times 10^{-5}$ & 101 \\
$c$ direction & $-3.261 \times 10^{-5}$ & -251 & $-0.380 \times 10^{-5}$ & -29 \\
\hline \hline
\end{tabular}

the hydrostatic compression needed to shift the maximum permittivity of, e.g., PMN-30PT or PLZT $6 / 65 / 35$ by $12{ }^{\circ} \mathrm{C}$ $[52,53]$, which is the maximum temperature difference between $T_{\mathrm{d}}$ and $T_{\mathrm{d}}^{*}$ as observed in the present study. At the surface, the chemical pressure vanishes and with it its stabilizing influence on the ferroelectric domain structure, leading to the observed lower depolarization temperature. As dopinginduced chemical pressure is a widely present phenomenon, its different impact on the bulk and surface properties should be observable in a broad variety of materials.

\section{CONCLUSION}

The achievable strain per unit electric field is one of the most important parameters for piezoelectric/ferroelectric applications. Materials showing a reversible relaxor-ferroelectric transition, as the one presented here, show exceptionally good performance in this regard, when driven above the transition temperature $T_{\mathrm{F}-\mathrm{R}}$, where the strain response is determined by the reversible transition between the polar and nonpolar phases. Below $T_{\mathrm{F}-\mathrm{R}}$ the continuous change in hysteresis shape and unipolar strain with increasing temperature (Fig. 1) points to the importance of the interplay between the two different phases present and their susceptibility towards the local mechanical stress state. The higher strains observed in this temperature regime might originate from the reversible detexturization of the tetragonal minority phase induced by the interfacial stresses between the two phases. This would make more ferroelastic domains available to contribute to the strain upon electric-field application. Furthermore, the regions with randomized domain structure can locally disrupt the ferroelectric domain alignment and promote the loss of coherency. The existence of such seeds could be the origin of the specific polarization reversal mechanism in the temperature range below $T_{\mathrm{F}-\mathrm{R}}$ that is specified by a nonpolar phase mediating the transition from one polar phase to the other upon electric-field reversal [8].

The presence of interfacial stresses between different crystallographic phases seems to be key for the performance of multiphase relaxor-ferroelectric systems. This opens up a door to tune their properties by controlled precipitation of optimized ratios of crystallographic phases. Tailoring of transition temperatures and stabilization/destabilization of ferroelectric domain structures can be targeted through this "in-build" stress. This is of high interest within the ferroelectric area for the development and optimization of actuator materials, piezotronics, as well as electrocalorics, and can be as well a useful tool to adjust metal-insulator transitions, stabilize metastable phases with desired functionality, or adjust transition temperatures in multiferroic materials.

\section{ACKNOWLEDGMENTS}

J.G. and J.S. acknowledge support by the Australian Research Council through Discovery Grants. M.H. acknowledges funding through the German Research Society DFG (Grant No. 1867/1-1) and M.A. acknowledges the support of the Feodor Lynen Research Fellowship Program of the Alexander von Humboldt Foundation. 


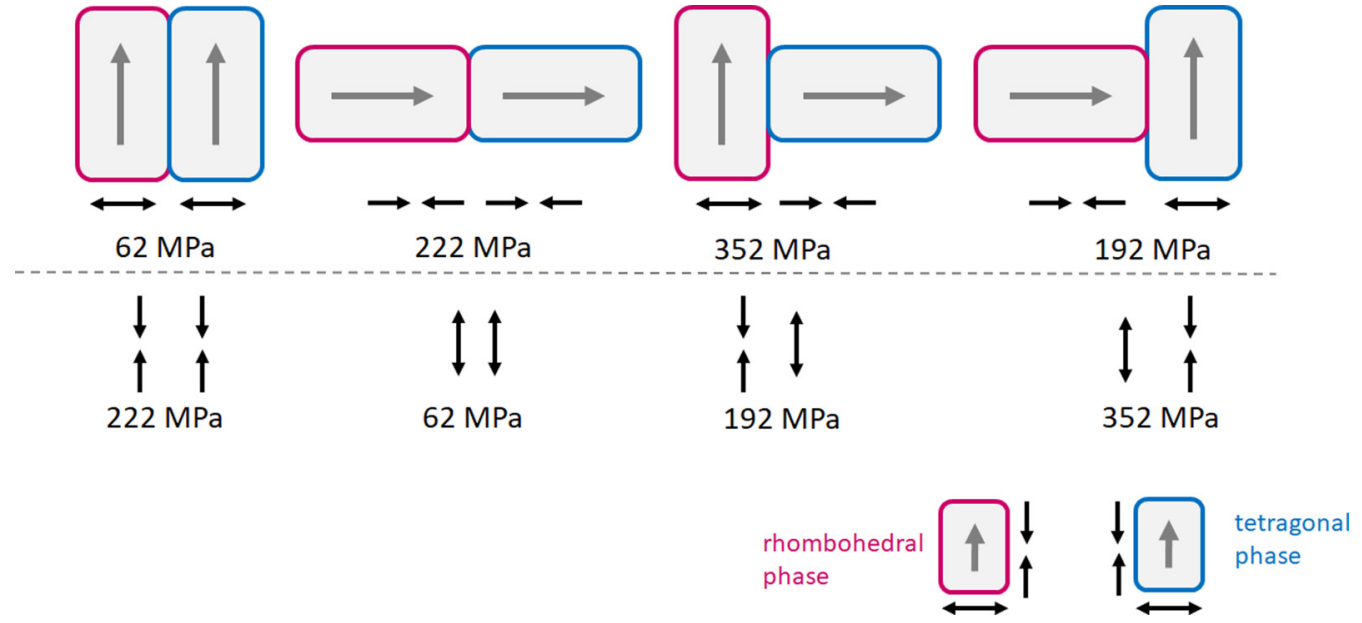

FIG. 7. Sketch of different phase orientations and the arising absolute interfacial stresses. The gray arrows inside the boxes indicate the $c$ direction for both phases; the black arrows indicate direction of expansion/compression.

[1] J. E. Daniels, W. Jo, J. Rödel, and J. L. Jones, Appl. Phys. Lett. 95, 032904 (2009).

[2] Z.-G. Ye, Key Eng. Mater. 155-156, 81 (1998).

[3] M. Hinterstein, M. Knapp, M. Hölzel, W. Jo, A. Cervellino, H. Ehrenberg, and H. Fuess, J. Appl. Crystallogr. 43, 1314 (2010).

[4] M. Acosta, L. A. Schmitt, C. Cazorla, A. Studer, A. Zintler, J. Glaum, H.-J. Kleebe, W. Donner, M. Hoffman, J. Rödel, and M. Hinterstein, Sci. Rep. 6, 28742 (2016).

[5] M. Hinterstein, L. A. Schmitt, M. Hoelzel, W. Jo, J. Rödel, H.-J. Kleebe, and M. Hoffman, Appl. Phys. Lett. 106, 222904 (2015).

[6] S.-T. Zhang, A. B. Kounga, E. Aulbach, H. Ehrenberg, and J. Rödel, Appl. Phys. Lett. 91, 112906 (2007).

[7] W. Jo, R. Dittmer, M. Acosta, J. Zang, C. Groh, E. Sapper, K. Wang, and J. Rödel, J. Electroceram. 29, 71 (2012).

[8] J. Glaum, H. Simons, J. Hudspeth, M. Acosta, and J. E. Daniels, Appl. Phys. Lett. 107, 232906 (2015).

[9] A. A. Bokov and Z.-G. Ye, J. Mater. Sci. 41, 31 (2006).

[10] A. A. Bokov and Z.-G. Ye, J. Adv. Dielectr. 2, 1241010 (2012).

[11] V. V. Shvartsman and D. C. Lupascu, J. Am. Ceram. Soc. 95, 1 (2012).

[12] W. Kleemann, J. Adv. Dielectr. 2, 1241001 (2012).

[13] V. Westphal, W. Kleemann, and M. D. Glinchuk, Phys. Rev. Lett. 68, 847 (1992).

[14] G. A. Samara, J. Phys.: Condens. Matter 15, R367 (2003).

[15] A. Bosak, D. Chernyshov, S. Vakhrushev, and M. Krisch, Acta Crystallogr., Sect. A 68, 117 (2012).

[16] V. Bobnar, Z. Kutnjak, R. Pirc, and A. Levstik, Phys. Rev. B 60, 6420 (1999).

[17] E. Sapper, N. Novak, W. Jo, T. Granzow, and J. Rödel, J. Appl. Phys. 115, 194104 (2014).

[18] W. Jo, J. Daniels, D. Damjanovic, W. Kleemann, and J. Rödel, Appl. Phys. Lett. 102, 192903 (2013).

[19] H. Simons, J. E. Daniels, W. Jo, R. Dittmer, A. J. Studer, M. Avdeev, J. Rödel, and M. Hoffman, Appl. Phys. Lett. 98, 082901 (2011).

[20] J. Glaum, H. Simons, M. Acosta, and M. Hoffman, J. Am. Ceram. Soc. 96, 2881 (2013).
[21] C. Groh, D. J. Franzbach, W. Jo, K. G. Webber, J. Kling, L. A Schmitt, H.-J. Kleebe, S.-J. Jeong, J.-S. Lee, and J. Rödel, Adv. Funct. Mater. 24, 356 (2014).

[22] N. Liu, M. Acosta, S. Wang, B.-X. Xu, R. W. Stark, and C. Dietz, Sci. Rep. 6, 36910 (2016)

[23] J. Zang, W. Jo, and J. Rödel, Appl. Phys. Lett. 102, 032901 (2013).

[24] F. H. Schader, Z. Wang, M. Hinterstein, J. E. Daniels, and K. G. Webber, Phys. Rev. B 93, 134111 (2016).

[25] G. Picht, K. G. Webber, Y. Zhang, H. Kungl, D. Damjanovic, and M. J. Hoffmann, J. Appl. Phys. 112, 124101 (2012).

[26] E. A. McLaughlin, T. Liu, and C. S. Lynch, Acta Mater. 52, 3849 (2004).

[27] M. G. Cain, S. M. Bennington, M. H. Lewis, and S. Hull, Philos. Mag. Part B 69, 499 (1994).

[28] F. Delogu, Acta Mater. 59, 2069 (2011).

[29] B. T. Lester, T. Baxevanis, Y. Chemisky, and D. C. Lagoudas, Acta Mech. 226, 3907 (2015).

[30] L. A. Schmitt and H. J. Kleebe, Funct. Mater. Lett. 03, 55 (2010).

[31] W. Jo, S. Schaab, E. Sapper, L. A. Schmitt, H.-J. Kleebe, A. J. Bell, and J. Rödel, J. Appl. Phys. 110, 074106 (2011).

[32] C. Ma and X. Tan, J. Am. Ceram. Soc. 94, 4040 (2011).

[33] D. I. Woodward, R. Dittmer, W. Jo, D. Walker, D. S. Keeble, M. W. Dale, J. Rödel, and P. A. Thomas, J. Appl. Phys. 115, 114109 (2014).

[34] B. Wylie-van Eerd, D. Damjanovic, N. Klein, N. Setter, and J. Trodahl, Phys. Rev. B 82, 104112 (2010).

[35] M. Acosta, J. Zang, W. Jo, and J. Rödel, J. Eur. Ceram. Soc. 32, 4327 (2012).

[36] A. J. Studer, M. E. Hagen, and T. J. Noakes, Phys. B 385-386, 1013 (2006).

[37] H. Simons, J. E. Daniels, A. J. Studer, J. L. Jones, and M. Hoffman, J. Electroceram. 32, 283 (2014).

[38] S. Matthies, L. Lutterotti, and H. R. Wenk, J. Appl. Crystallogr. 30, 31 (1997).

[39] M. Hinterstein, M. Hoelzel, J. Rouquette, J. Haines, J. Glaum, H. Kungl, and M. Hoffman, Acta Mater. 94, 319 (2015). 
[40] See Supplemental Material at http://link.aps.org/supplemental/ 10.1103/PhysRevMaterials.3.054406 for additional dielectric, microstructural, and crystallographic details.

[41] M. Vögler, N. Novak, F. H. Schader, and J. Rödel, Phys. Rev. B 95, 024104 (2017).

[42] D. Gobeljic, R. Dittmer, J. Rödel, V. V. Shvartsman, and D. C. Lupascu, J. Am. Ceram. Soc. 97, 3904 (2014).

[43] J. Glaum, M. Zakhozheva, M. Acosta, E. Aksel, H.-J. Kleebe, M. Hoffman, and L. A. Schmitt, J. Am. Ceram. Soc. 99, 2801 (2016).

[44] J. Kling, W. Jo, R. Dittmer, S. Schaab, and H.-J. Kleebe, J. Am. Ceram. Soc. 96, 3312 (2013).

[45] C. Ma, H. Guo, S. P. Beckman, and X. Tan, Phys. Rev. Lett. 109, 107602 (2012).

[46] M. Deluca, G. Picht, M. J. Hoffmann, A. Rechtenbach, J. Töpfer, F. H. Schader, and K. G. Webber, J. Appl. Phys. 117, 134110 (2015).
[47] Y. Heo, B.-K. Jang, S. J. Kim, C.-H. Yang, and J. Seidel, Adv. Mater. 26, 7568 (2014).

[48] P. Sharma, Y. Heo, B. Jang, Y. Liu, V. Nagarajan, J. Li, C. Yang, and J. Seidel, Adv. Mater. Interfaces 3, 1600033 (2016).

[49] K. G. Webber, Y. Zhang, W. Jo, J. E. Daniels, and J. Rödel, J. Appl. Phys. 108, 014101 (2010).

[50] S. M. Denkhaus, M. Vögler, N. Novak, and J. Rödel, J. Am. Ceram. Soc. 100, 4760 (2017).

[51] E. Sapper, S. Schaab, W. Jo, T. Granzow, and J. Rödel, J. Appl. Phys. 111, 014105 (2012).

[52] A. A. Bokov, A. Hilczer, M. Szafránski, and Z. G. Ye, Phys. Rev. B 76, 184116 (2007).

[53] G. A. Samara, Ferroelectrics 274, 183 (2002).

[54] I. S. Vorotiahin, E. A. Eliseev, Q. Li, S. V. Kalinin, Y. A. Genenko, and A. N. Morozovska, Acta Mater. 137, 85 (2017).

[55] S. Trujillo, J. Kreisel, Q. Jiang, J. H. Smith, P. A. Thomas, P. Bouvier, and F. Weiss, J. Phys.: Condens. Matter 17, 6587 (2005). 